\title{
AvaliaÇÃo assistida e COMUNiCAÇÃo alternativa: PROCEDIMENTOS PARA A EDUCAÇÃO INCLUSIVA ${ }^{1}$ \\ Dynamic assessment and augmentative and alternative \\ COMMUNICATION: PROCEDURES FOR INCLUSIVE EDUCATION
}

Kely Maria Pereira dePAULA²
Sônia Regina Fiorim ENUMO

RESU M O : as pesquisas sobre a avaliação assistida vêm delineando um campo promissor dada as suas características inovadoras que a distinguem de uma avaliação tradicional, favorecendo a população que apresenta dificuldades no processo de aprendizagem ou em situação de desvantagem social. Crianças que necessitam de recursos al ternativos para a linguagem expressiva constituem um significativo grupo que pode sebeneficiar deuma avaliação diferenciada. Nesteestudo, participaram setecrianças comnecessidades especiais em situação de avaliação assistida, utilizando oC hildren's A nal ogical Thinking M odifiability Test-CATM, acrescida da avaliação psicométrica, realizada pelos testes Raven, Columbia e Peabody, estes últimos em versão computadorizada, antes e após intervenção com sistema deComunicação A Iternativa eA mpliada-CAA. A lém dos processos de pensamento, foram considerados aspectos não estritamente cognitivos, mas de grande importância: reação da criança ao contexto de aval iação e ao tipo detarefa, variáveis pessoais esituacionais como motivação efadiga, além da acessibilidadeà mediação. Tais variáveis foram sistematizadas em categorias cognitivas, comportamentais e afetivo-motivacionais nos contextos de ensino eintervenção, compondo um mosaico deindicadores sobre o perfil da amostra nas diferentes etapas do estudo. A pesar da situação deavaliação - tradicional eassistida - ter se configurado como cansativa, foi possível identificar as operações e disfunções cognitivas nas provas tradicionais eassistida ena intervenção, além defatores não-intel ectuais que afetaram o desempenho, delineando um perfil intragrupo de aprendizagem na resolução de problemas. A avaliação assistida éreconhecidamenteuma relevanteferramenta dediagnóstico psicológico, complementar à abordagem psicométrica tradicional, para crianças com déficits comunicativos.

PA LA V RA S-CH A V E: aval iação assistida; comunicação al ternativa eampliada; crianças com problemas de comunicação; educação especial.

\footnotetext{
${ }^{1}$ Estudo derivado da tese de doutorado, realizada pela primei ra autora sob orientação da segunda, defendida no Programa de Pós-Graduação em Psicologia-PPGP, UFES. A s autoras agradecem a todos os integrantes do grupo de pesquisa pelas discussões teóricas, às bolsistas de Iniciação Científica pela partici pação na col eta de dados eàs agências de fomento pelo auxílio financeiro, entre elas, CA PES (bolsa de doutorado), CN Pq (Proc. n 520808/ 97-5: bolsas de IC e PIBIC) e Prefeitura Municipal de Vitória (FACITEC Proc. n5012579/ 2001: bolsa de $A$ perfeiçoamento eauxílio à pesquisa).

${ }^{2}$ Doutora em Psicologia pela Universidade Federal do Espírito Santo - UFES, Professora-Adjunta do Departamento de Psicologia Social edo Desenvolvimento e do Programa de Pós-Graduação em Psicologia da UFES. Endereço para correspondência: Universidade Federal do Espírito Santo, Departamento de Psicologia Social e do Desenvolvimento. A v. Fernando Ferrari, 514, campus universitário "Alaor de Queiroz Araújo", Centro de Ciências Humanas eN aturais, Bairro Goiabeiras, Vitória, ES CEP 29075-910- kelympp@terra.com.br. ${ }^{3}$ Doutora em Psi cologia pela UniversidadedeSão Paulo - USP, com pós-doutorado na U niversidadeEstadual deCampinas - UNICAMP, Professora-A djunta do Departamento de Psicologia Social edo Desenvolvimento e do Programa de Pós-Graduação em Psicologia da Universi dadeFederal do Espírito Santo - UFES. Endereço para correspondência: UniversidadeFederal do Espírito Santo, Departamento de Psicologia Social edo Desenvolvimento. A v. Fernando Ferrari, 514, campus universitário "Alaor de Queiroz Araújo", Centro deCiências Humanas e N aturais, Bairro Goiabeiras, Vitória, ES CEP 29075-910 - soniaenumo@terra.com.br.
} 


\begin{abstract}
A BST RACT : research on dynamic assessment points to a promising field where its innovative features differ from traditional assessment by favoring the population that presents challenges in the learning processes or who present socially disadvantaged conditions. Children who need alternativeresources for expressivelanguage constitute a significant group that can be benefited from assessment tailored to their needs. In this study, seven children with special needs (communication problems) took part in a dynamic assessment situation using Tzuriel and Klein's Children's A nalogical Thinking Modifiability Test (CATM), in order to complete the psychometric assessment carried out by Raven, Columbia and TVIP tests. Thelatter was a computerized version, applied before and after intervention with an Augmentative and AlternativeCommunication (AAC) system. Besides the thought processes, other not specifically cognitive aspects of great importance were considered: thechild's reaction to the assessment situation and the kind of task put before, personal and situational variables such as motivation and fatigue, as well as accessibility to the mediation strategies. Such variables were systematized in cognitive, behavioral and affective-motivational categories in the teaching and intervention contexts compounding a mosaic of indicators about the characteristics of children with communicative deficits at different stages of thestudy. Even though the assessment conditions - traditional and dynamic - weretiring for the children, wewere able to identify the operations and cognitivedysfunctions both in thetraditional tests and in the dynamic testing, as well as in theintervention. Furthermore, affectivemotivational and behavioral factors were shown to affect performance, composing an intra-group learning profile for problem solving. Thus, the study demonstrated that dynamic assessment can be an important tool for psychological diagnosis, complementing traditional psychometric approach, for children with communication problems.
\end{abstract}

KEYWO RD S: dynamic assessment; augmentativeand alternative communication; children with communication problems; special education.

\title{
InTRODUÇÃo
}

A Avaliação Assistida, assim como a Comunicação Alternativa e A mpliada, são temas de investigação recentes, incrementados no país pelas pesquisas desenvolvidas em centros universitários a partir da década de $90 . \mathrm{Na}$ integração dessas duas áreas, que em comum convergem para um melhor desenvolvimento das pessoas com necessidades especiais, houve, em nosso estudo, o desafio metodológico de verificar se o procedimento assistido, com a aplicação deseus instrumentos diferenciados, ésensível para avaliar deforma mais amplae prescritiva, crianças que apresentam déficits comunicativos. Para tanto, a teoria de Vygotsky, mais particularmente o conceito de Zona de Desenvolvimento Proximal eo conceito de Experiência de A prendizagem M ediada deFeuerstein se constituíram no aporte teórico quefundamentou tais aplicações.

Mesmo que a linguagem verbal não seja a única modalidade de comunicação, quando o repertório verbal éinadequado, ou seja, quando a fala não se manifesta, éininteligível ou sobremodo limitada, reduz-se consideravelmentea oportunidade de interação face a face nos diversos ambientes sociais. Para essa população, são necessários dispositivos - sistemas de Comunicação A Iternativa e A mpliada (CAA ) - que permitem uma comunicação significativa e mais funcional no contexto sócio-afetivo.

A CAA tem sido comumente caracterizada como uma área da prática clínica que visa compensar, temporária ou permanentemente, desordens na comunicação expressiva, dado os prejuízos nalinguagem (oral eescrita). Diferentes meios de comunicação derivados do uso degestos, linguagem desinais eexpressões faciais, figuras, símbolos, além de sofisticados sistemas computadorizados podem 
ser empregados deforma substitutiva ou suplementar de apoio à fala, ajudando a desenvolver, quando possível, a linguagem oral (NUNES, 2003).

No Brasil, o emprego daCAA foi iniciado em instituições dereabilitação para portadores de deficiência física, especialmente paral isia cerebral. N os últimos anos, houve um interesse crescentepela área com o estudo de temas como: recursos multimídia para aval iação de habilidades cognitivas em pessoas com necessidades especiais (CAPOVILLA; THIERS; CAPOVILLA, 2003); estratégias de ensino naturalístico para implementação e desenvolvimento da linguagem alternativa (NUNES, 2003; PA ULA , 1998); anál ise psicolingüística da comunicação simbólica (NUNES et al., 2000), entre outros. De forma ampla, os resultados atestam que para a aquisição e general ização de habilidades de comunicação alternativa por indivíduos com déficits lingüísticos é necessário que haja, sobretudo, mudanças sistemáticas nos ambientes sociais nos quais eles estão inseridos.

Considerando a proposta de inclusão de crianças com necessidades educacionais especiais (NEE) no sistema regular de ensino (OMOTE, 2004; STAINBACK; STAINBACK, 1999), épreciso quesejam empregadastanto medidas de avaliação quanto estratégias de intervenção eficazes na promoção das habilidades defasadas. Atrelada à questão da avaliação psicológica, além da estigmatização de pessoas com deficiência, demais críticas teóricas emetodológicas vêm sendo levantadas desde a década de 60 do século passado sobre a aplicação de instrumentos de medida de inteligência, entre elas: o uso indiscriminado dos testes de QI e a tomada de seus resultados como absolutos, a adoção de uma concepção inatista sobre a origem das diferenças individuais, a ausência de "neutralidade social" com grupos minoritários e a sua inadequação aos demais grupos culturais (STERNBERG; GRIGORENKO, 2002). Assim, concordamos com a concepção mais contemporânea de que o QI não é sinônimo da capacidade de aprender, ou seja, o conceito de inteligência, entendido como uma "habilidade inata", não é suficiente para explicar as diferenças individuais no pensamento, percepção, aprendizagem, solução de problemas einteração social (LIDZ, 1991).

Em meio ao debate acalorado sobre os instrumentos de medida de inteligência, marcado basicamente pela aplicação de testes psicométricos tradicionais, surgiu, na década de 70, em Israel e nos Estados Unidos, uma abordagem al ternativa de avaliação quefornecia indicadores sobre o potencial do indivíduo para aprender denominada avaliação assistida, "dinâmica" ou "interativa", sendo utilizada de forma substitutiva ou complementar à abordagem psicométrica(ENUMO, 2005; LIDZ, 1991; LINHARES; ESCOLANO; ENUMO, 2006; STERNBERG; GRIGORENKO, 2002; TZURIEL, 2001).

A emergência desse novo modelo foi parcialmente motivada pela crescente insatisfação com as informações restritas fornecidas sobrea relação entre desenvolvimento psicológico e progresso escolar das crianças, quase que exclusivamenteobtidas através das abordagens psicométricas. Tal insatisfação se traduziu em uma nova proposta que necessariamente deveria produzir: uma 
medida direta do potencial de aprendizagem e do nível de desenvolvimento; informações sobre os processos de pensamento que levam a criança a se desempenhar deforma eficiente ou deficitária em tarefas cognitivas; eindicações sobre as estratégias que facilitam a educação e a superação de problemas no desenvolvimento. Em outras palavras, a avaliação assistida é definida como um procedimento deavaliação do pensamento, percepção, aprendizagem e resolução de problemas, mediante um processo deensino quevisa modificar o funcionamento cognitivo, sendo uma abordagem ativa à deficiência e às dificuldades de aprendizagem (FON SECA, 1998; TZURIEL, 2001).

Essa inovadora abordagem de avaliação tem como fundamentação teórica o conceito amplamente discutido na atual idade deZ ona de $D$ esenvol vimento Proximal - ZDP, deVygotsky (1991), eo conceito de Experiência deA prendizagem Mediada - EAM, deFeuerstein, ambos trazendo destaque para o papel das variáveis sócio-culturais no desenvolvimento cognitivo (FEUERSTEIN et al., 1987; TZURIEL, 2001).

A conceção de Modificabilidade Cognitiva Estrutural - MCE, também desenvolvida por Feuerstein ao final da década de 50, é outro pilar da avaliação assistida. Historicamente, a teoria da MCE surgiu de sua prática como psicólogo clínico educacional e pesquisador da cognição (GOMES, 2002). Na situação do pós-guerra havia um quadro generalizado de "desolação" nos ambientes educacionais decorrente das precárias condições "psicológicas" de crianças e adolescentes israelenses que sobreviveram ao horror do Holocausto. Partindo de uma concepção mais "otimista" acerca da natureza humana, Feuerstein visualizou para além da aval iação psicométrica e considerou que essas crianças tinham um potencial que os testes convencionais não conseguiam evidenciar.

\begin{abstract}
Lidar com crianças nessas terríveis condições de vida confrontou-me com a questão profética de Ezeklian: “De al gum modo, esses ossos viverão?" Será possível reverter o resultado das mais difíceis condições em queessas crianças viveram ao longo de sua infância e adolescência nos campos de morte do H olocausto? [...] A ssim, em lugar de buscar por fontes teóricas queservissem de base para uma visão otimista do ser humano e da possibilidade demudar o curso desuavida, buscamos meios deprovar queisso era possível, envolvendonos em um programa de diagnóstico eintervenção a fim de confirmar nosso postulado e nosso sistema otimista de crença na modificabilidade de sua condição: cognitiva, emocional ou comportamental. (FEUERSTEIN ; FEUERSTEIN, apud GOMES, 2002, p. 14)
\end{abstract}

A pós uma intervenção com ensino al tamente focalizado etratamento psicológico, em formato simples do tipo "testagem-ensino-testagem", houve mudança nas sombrias estatísticas: muitos que estavam nas classificações mais rebaixadas alcançaram níveis normais de desenvolvimento, enquanto outros chegaram à universidadeeàs profissões acadêmicas. Desdeentão, muitos estudos empíricos foram desenvolvidos a partir desses princípios (FEUERSTEIN et al., 1987; TZURIEL, 2001). 
Em um dos poucos estudos que integra as concepções de Feuerstein, como as teorias da MCE e da EAM à aplicação de sistemas alternativos de comunicação, Carrillo e Thompson (2002) avaliaram crianças não-verbais e com baixo desempenho comunicativo objetivando identificar e modificar as funções cognitivas deficitárias. Utilizando o formato "pré-mediação e pós-mediação" para análise de diferentes funções cognitivas (planejamento, comparação, e categorização, por exemplo), as autoras consideraram que as estratégias mais eficientes para modificar o funcionamento das crianças foram: generalização (transferência), permanência (persistência) e centralidade (mediação para autonomia eautoregulação).

Sendo uma proposta holística, além da mediação, a avaliação assistida contempla aspectos não estritamente cognitivos como os fatores emocionais e motivacionais que podem determinar o estilo de aprendizagem da criança e de seu sucesso acadêmico (TZURIEL; SA MUELS, 2000). Para analisar a relação entre os aspectos não intelectuais e a aprendizagem, Tzuriel (2001) eTzuriel, Samuels e Feuerstein (1988) apontaram sete fatores que devem ser considerados durante o procedimento assistido na interação mediador-mediado: acessi bilidade à mediação; necessidade de domínio (persistência emotivação intrínseca à tarefa são conceitos relacionados); tolerância à frustração; locus decontroleinterno (percepção deser responsável pelo resultado de seu comportamento e controle sobre os eventos da vida); medo de fracassar e atitude defensiva; confiança na resposta correta; e vitalidade e sentido de alerta (nível de atividade, vivacidade e interesse que a criança demonstra na interação com o mediador).

Seguindo a tendência de pesquisas realizadas em outros países, desde a década de 90, estamos presenciando um crescente interesse pela avaliação assistida no Brasil envolvendo crianças com dificuldades de aprendizagem (LINHARES et al., 1998), deficiência mental (SANTA MARIA ; LINHARES, 1999), visual (BATISTA; HORINO; NUNES, 2004; ENUMO; BATISTA , 2000) eproblemas de comunicação (PAULA; ENUMO, no prelo). Esse procedimento também está sendo utilizado para avaliar: os efeitos dos programas de intervenção em criatividade e sistemas de CAA (DIAS; ENUMO, 2006; DIAS et al., no prelo); a influência dos fatores não-intelectuais em provas assistidas para crianças com NEE (ENUMO et al., 2003; PAULA, 2004); a mediação do examinador em provas assistidas (ENUMO et al., 2002), entreoutras temáticas importantes(ENUM O; 2005; LINHARES; ESCOLANO;ENUMO, 2006).

Entre os principais resultados, podemos destacar a melhora no desempenho após a mediação, dependendo do tipo e intensidade do ensino, da natureza das barreiras cognitivas e da distância psicológica entre o conteúdo de ensino e o conteúdo de desempenho dos testes. Há ainda a transferência das estratégias mediadas para novos contextos deresolução deproblemas. Isto gera uma medida do potencial de aprendizagem que, de outro modo, seria difícil obter em uma testagem normativa, como ocorre, por exemplo, na avaliação de examinandos 
que resistem à avaliação, que possuem graves problemas de comunicação e que possuem barreiras psicológicas (por exemplo, o autoconceito negativo como aprendiz) para a expressão das habilidades requeridas em tarefas cognitivas. É um procedimento quefornece previsões mais otimistas do queaquelas produzidas por meio dos testes estáticos (HAYWOOD; TZURIEL, 2002; TZURIEL, 2001).

A pesar dos relevantes resultados, críticas são levantadas sobre a extensão em que esses dados podem ser generalizáveis: a) há poucos estudos empíricos; b) persistem os problemas psicométricos, como a questão da fidedignidade (maior no formato estático, ou seja, sem mediação); c) a validade ainda precisa ser obtida; d) o modelo não está incluído de forma consistente no campo da avaliação psicológica e, consequentemente, o tema ainda não éabordado em cursos de graduação, sendo a divulgação incipiente; e) a aplicação demanda mais tempo; ef) ainda há a baixa concordância entrejuízes na determinação das funções cognitivas deficitárias que requerem mediação, no tipo e quantidade de mediação a ser empregada ena interpretação das di ferenças de desempenho antes e após a mediação (STERNBERG; GRIGORENKO, 2002). Sobreesteúltimo ponto, embora poucos, estudos têm sido conduzidos com o objetivo de medir o nível de concordância entre juízes, especialmente na avaliação das funções cognitivas deficientes, tipos de mediação einfluência dosfatores não-intelectuais (TZURIEL; SAMUELS, 2000; VAUGHT;HAYWOOD, 1990).

Vygotsky contestou as idéias organicistas, vigentes em sua época, que reduziam a deficiência a aspectos puramente biológicos. Sua concepção sobre a deficiência pode ser considerada extremamentemoderna para a Rússia do início do século XX ao propor uma abordagem centrada na superação do déficit orgânico. A tual mente, reconhece-se como a deficiência secundária, vinculada ao conceito de incapacidade (disability) e de desvantagem (handicap), impede o desenvolvimento, aprisionando a pessoa em uma rede de significações sociais pautadas por atitudes, preconceitos e estereótipos que perpetuam sua condição estigmatizada, qual seja, a de ser portadora de uma diferença em uma sociedade de "normais" (Omote, 2004). Todavia, o desenvolvimento da CAA e de outras práxis da Psicologia como a avaliação assistida, têm possibilitado diminuir as barreiras da exclusão social por meio de al ternativas que melhorem a qualidade de vida da pessoa deficiente e, conseqüentemente, seu processo de inserção na cultura.

Da interface teórico-prática das áreas aqui descritas o seguinteobjetivo emergiu: anal isar a ad equação da avaliação assisti da para crianças com problemas de comunicação, comparando o seu desempenho em provas psicométricas tradicionais e prova assistida, fornecendo indicadores dos efeitos de um programa de intervenção com sistema alternativo de comunicação sobre as habilidades lingüísticas e cognitivas dessas crianças. 
Método

Participantes e local

Participaram setecrianças, ${ }^{4}$ com idades entre 8 e 11 anos, freqüentando entre a 1a e 3a série do Ensino Fundamental de escolas pública e particular da cidade deVitória e região serrana do ES, portadoras de NEE edéficits quevariavam de moderado a severo nas habilidades delinguagem ecomunicação, decorrentes deetiologias diversas, entreelas, síndromedeDown, deficiência mental, tetraplegia mista, meningite e atraso generalizado no desenvolvimento, sem diagnóstico definido.

A pesquisa foi conduzida em duas escolas da capital e também no Laboratório de Desenvolvimento eA prendizagem do Departamento dePsicologia Social e do Desenvolvimento da UFES durante 11 meses, entre os anos de $2002 \mathrm{e}$ $2003^{5}$

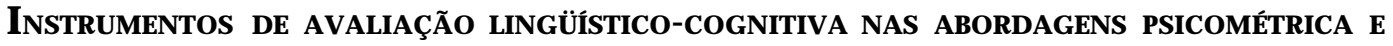 ASSISTIDA}

Para uma caracterização inicial da amostra foram utilizadas entrevistas com as mães e com as educadoras das escolas freqüentadas. Na avaliação da comunicação aplicamos a Escala de Comunicação Pré-Verbal - ECPV (KIERNAN; REID, 1988, apud PAULA, 2004), a Lista de A val iação do Vocabulário Expressivo - LAVE (RESCORLA, 1989, traduzida por CA POVILLA; CAPOVILLA, 1997) eum Protocolo de A valiação da Comuni cação adaptado (PA ULA , 2004) com o objetivo delevantar a extensão do repertório comunicativo, tanto verbal quanto não-verbal.

Entre os instrumentos de avaliação lingüístico-cognitiva incluídos em uma abordagem psicométrica utilizamos a Escala Columbia de Maturidade Intelectual (RODRIGUES; ROCHA, 1999) e o Teste de Vocabulário por Imagens Peabody - TVIP (CAPOVILLA; CA POVILLA, 1997), ambos em versão computadorizada(CA POVILLA; DUDUCHI; MACEDO, 1997a), alémdas Matrizes Progressivas Coloridas deRaven - Escala Especial (RAVEN, 1974, apud ANGELINI et al., 1999) para medidas do nível intelectual geral eda linguagem receptiva.

Gostaríamos de justificar, além da avaliação assistida, o uso de uma abordagem psicométrica. Embora a literatura mostre seguidamente baixas classificações em instrumentos padronizados na população especial (WARREN, 1994), o uso de provas tradicionais se justificou, primeiro, pela adoção do

\footnotetext{
${ }^{4} \mathrm{~A}$ aceitação foi formalizada mediante a assinatura, pelo responsável, do Termo de Consentimento para partici pação em pesquisa, segundo normas estabelecidas pel o M inistério da Saúdee pela Resolução n 016/2000 do Consel ho Federal dePsicologia, tendo o projeto sido aprovado pelo Comitêde Ética do Programa de PósGraduação em Psicologia da UFES. Para preservação da identidadefaremos referência aos partici pantes por nomes fictícios: I gor, Wellington, Marcus, Guilherme, Vinícius, Fabrícia eFernanda.

${ }^{5}$ Foi utilizado um del ineamento em queo sujeito serve como seu próprio controle, antes eapós um período de intervenção (MELTZOFF, 2001).
} 
procedimento de avaliação assistida estruturado, comumente utilizado em pesquisa, de forma complementar e não como modelo substituto da avaliação estática (LIDZ, 1991; TZURIEL, 2001). Em segundo lugar, ainda vinculado à primeira questão, atéo momento da coleta de dados, não havia outros estudos no país que utilizassem esse procedimento em crianças com problemas de comunicação ou com usuários deCAA.

Para a avaliação no formato assistido foi empregado o Children's A nalogical Thinking M odifiability Test - CATM (Teste de Modificabilidade do Pensamento A nalógico em Crianças), elaborado por Tzuriel e Klein (1990) para pré-escolares ou crianças com NEE, avaliando o raciocínio analógico (A:B::C:?) na resolução de problemas. São 18 blocos coloridos e três conjuntos de problemas analógicos querequerem reconhecimento detrês dimensões: cor, forma etamanho. É preciso inferir a relação entre os dois primeiros componentes da analogia $(A: B)$ e aplicá-la aos outros dois (C:D), sem necessariamente emitir resposta verbal para resolução. Há quatro níveis de dificuldade. No nível 1 uma dimensão muda; no nível 2 mudam duas dimensões. Todas as três dimensões semodificam nos níveis 3 e 4, mas, neste último, há uma novidade: é preciso estabelecer ainda uma correlação entre o bloco superior e o inferior no primeiro par da analogia, transferindo-a ao segundo par. A Figuras 1 e a Figura 2 exemplificam cartões do CATM, sendo os respectivos blocos colocados sob cada uma dos componentes da analogia.

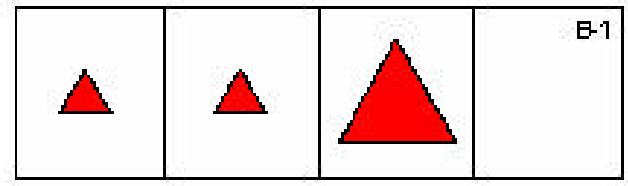

Figura 1. Primeiro cartão da fase de Manutenção. Uma dimensão muda: tamanho (Nível 1).

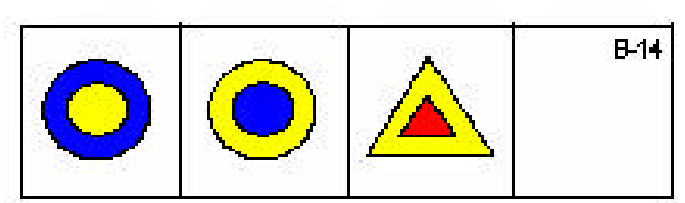

Figura 2. Último cartão da fase de Transferência. Todas as dimensões mudam nos blocos superpostos (Nível 4).

O CATM foi organizado em cinco fases: preliminar, sem ajuda, assistência, manutenção etransferência (SANTA MARIA; LIN HARES, 1999) ${ }^{6}$. Na fase preliminar (PRE) a criança deve montar uma matriz, classificando os blocos segundo seus atributos. A fase sem ajuda (SAJ) serve como linha-de-base para avaliação do raciocínio analógico, por isso, nenhuma ajuda é oferecida para a resolução dos problemas. N a fase de assistência (ASS) ensina-se à criança a ater-se às dimensões relevantes para a resolução; compreender regras detransformação e princípios analógicos; procurar sistematicamente blocos corretos; e melhorar o desempenho. Na fase demanutenção (MAN) a suspensão da ajuda permite avaliar o desempenho comparado-o ao da fase sem ajuda (SAJ), ou seja, permite uma

\footnotetext{
${ }^{6}$ Santa M aria e Linhares (1999) aplicaram o CATM com modificações no teste original (TZURIEL; KLEIN, 1990), desl ocando os problemas com maior nível de dificuldade, apresentados ao final detodas as fases, para uma única fase, transferência, para verificar a general ização do desempenho em nova situação.
} 
medida do nível demodificabilidadecognitiva. A fasedetransferência (TRA), com ajuda parcial do mediador (indicando erros, mas sem conduzir à resposta correta), verifica se a criança generaliza habilidades adquiridas para uma situação mais complexa ${ }^{7}$.

Para avaliar sea criança mel hora o desempenho a partir da assistência do mediador são computados os acertos totais (crédito total) e parciais (crédito parcial ), quando a criança écapaz de resolver corretamente uma ou duas das três dimensões requeridas. Ainda, são estabelecidas classificações sobre o perfil de desempenho. ${ }^{8}$

O Protocolo de avaliação das operações cognitivas apresentou polaridades mutuamente exclusivas: conduta reflexiva ou impulsiva; percepção clara ou confusa; percepção integrativa ou episódica; conduta comparativa ou nãocomparativa; identificação de relevância ou dificuldade de identificação de relevância; autocorreção ou ausência de autocorreção e generalização ou ausência degeneralização (DIAS; ENUMO, 2006).

Deforma conjunta, outra escala bipolar foi empregada- Protocolo de avaliação do comportamento - considerando 11 comportamentos quanto a: sossegado ou inquieto, relaxado ou tenso, reflexivo ou impulsivo, participativo ou retraído, interessado ou desinteressado, concentrado ou disperso, orientado ou confuso, cuidadoso ou descuidado, persistente ou não-persistente, disposto ou cansado erápido ou lento (SANTA MARIA ; LINHARES, 1999).

Por fim, para anal isar a influência dos aspectos afetivo-motivacionais, nove fatores foram considerados: acessibilidade à mediação, necessidade de explorar a tarefa (curiosidade), prazer na atividade, trabalhar de forma independente, necessidadedecontinuar a trabal har em outras tarefas, tolerânciaà frustração, vitalidade e sentido de alerta, medo de falhar e atitude defensiva (TZURIEL, 2001).

\section{Materiais e instrumentos da intervenção em CAA}

Na fase deintervenção em habilidades de comunicação e pensamento utilizou-se como recurso facilitador o sistema computadorizado Imagoanavox (CAPOVILLA; DUDUCHI; MACEDO, 1997b) personalizado (inserção defotos, figuras e vocabulários significativos para cada criança). Um feedback (voz

\footnotetext{
${ }^{7}$ Esquema deaplicação das fases do CATM : PRE - matriz deblocos ecartão-exemplo; SA J - 6 cartões; ASS- 10 cartões; MAN - 6 cartões; eTRA - 2 cartões-exemplo e 10 cartões-problema.

${ }^{8}$ Perfis de desempenho: al to-escore- soluções analógicas eficientes na proporção de acertos de 0,75 ou mais na fase inicial SAJ e, no mínimo, 0,60 de acertos na fase de MAN ; ganhador - melhora o desempenho na fase deASS e o mantém, pelo menos, na proporção de 0,50 de acertos após a suspensão da ajuda; não-manten edor - não há mel hora na fase deASS comparada à fase ini cial SAJ ou apresenta melhora em uma proporção abaixo de 0,50 de acertos na fase deASS, que não se mantém na fase de MAN; transferidor - apresenta bom desempenho na fase de TRA, na proporção de 0,60 de acertos; enão-transferidor - apresenta menos de 0,60 deacertos na fase deTRA .
} 
digital izada) era fornecido a cada item acionado pel o mouse ou dispositivo adaptado (mediante varredura). Para facilitar as modalidades de comunicação, as mesmas fotos e figuras foram dispostas em um Fichário de comunicação (PAULA, 1998). A mbos os sistemas foram compostos por pictogramas do Picture Communication Symbols - PCS(JOHNSON, 1981, apud NUNES, 2003) por sua alta iconicidade, isto é, os ícones são facilmente interpretados como representantes dos respectivos referentes. Para ampliar a comunicação utilizamos materiais lúdicos epedagógicos como livros, cartões e fotos da criança e sua família para descrição dos eventos cotidianos.

Demais atividades foram realizadas no formato assistido como a prova deExclusão deObjetos (ENUMO; BATISTA , 2000) eo Jogo de A nalogia deFiguras. A primeira envolve habilidades de classificação ea segunda, baseada no subteste de analogia conceitual deTzuriel (2001), The Children's Conceptual and Perceptual A nalogies M odifiability Test, avalia o raciocínio analógico em uma matriz $2 \times 2$ $(A: B:: C: D)$.

\section{Resultados}

Para uma análise mais abrangente do estudo serão descritos os principais resultados. A análise dos dados obtidos por meio dos instrumentos específicos para avaliação da comunicação (ECPV, LAVE e Protocolo de Comunicação) indicou que a maioria das crianças possuía repertório lingüístico restrito, utilizando palavras "soltas" com funções comunicativas diversas (holófrases), sentenças simples de 3-4 palavras, combinadas com gestos, expressões faciais e vocalizações. Duas crianças, W el lington el gor , com melhor desempenho comunicativo no grupo, conseguiam construir al gumas sentenças bem elaboradas, com perguntas, respostas, solicitações e comentários simples. Contudo, havia comprometimento na função declarativa quando as sentenças apresentavam estrutura gramatical mais reduzida, com omissões de palavras-chaves e repetição desentenças.

\section{Pré-teste}

Houve uniformidade no desempenho geral da amostra nas provas psicométricas. Os resultados obtidos no Columbia indicaram níveis de raciocínio intelectual geral abaixo daidade(3a6m-3a11m). Desempenho semel hantefoi obtido no Raven-MPC (classificação deficiente), indicando extrema dificuldade na resolução de problemas baseados na percepção diretaeno raciocínio por inferências. No TVIP (vocabulário receptivo) o desempenho da maioria indicou idades equivalentes inferiores (2a6m-2allm).

Os comportamentos foram classificados em facilitadores enão-facilitadores para a execução da tarefa. N o pré-teste, o TVIP computadorizado foi a tarefa de melhor execução, com maior interesse, concentração e participação detodo o grupo. 
Comportamentos não-facilitadores ocorreram com maior freqüência no Columbia eno Raven-MPC, sendo esteúltimo a tarefa de execução mais difícil.

Considerando o desempenho na avaliação assistida, funções cognitivas deficitárias, comportamentos e fatores afetivo-motivacionais não facilitadores também dificultaram a execução do CATM, para a mai oria, em suas fases iniciais (dificuldades de manter constância nos atributos, impulsividade, baixa tolerância à frustração e esquiva.

W ell ington apresentou o melhor desempenho, realizando integral mente a prova assistida em um total de 3 sessões. Comportamentos não-facilitadoresforam mais freqüentes na fase de ASS $(0,91)$ que se tornou longa e cansativa devido à elevada freqüência de pistas para conduzir a criança à sol ução analógica (em média, 14,5dicas/ cartão). ${ }^{9}$ O padrão demediação da examinadora foi predominantemente do tipo instrução analítica (descrição e anál ise dos atributos do objeto) e feedback analítico (reforço de acertos e correção de atributos incorretos). A despeito das dificuldades cognitivas, a criança apresentou sol ução correta em 2 cartões logo na primeira tentativa, depois que se auto-regulou emantevea atenção, ol hando para o cartão e procurando o bloco correto (isto descartou uma sol ução do tipo correta ao acaso). Ainda que fatores comportamentais e motivacionais tenham comprometido a execução do CATM, W ellington apresentou desempenho um pouco melhor após a fase de ASS, sendo classificado no perfil ganhador, contudo, não estabeleceu generalização das habilidades para a fase de TRA (perfil nãotransferidor ). A criança obteveuma taxa deganho de 51,5\%, considerando a diferença entre as fases SAJ-MAN.

Quando se analisa o crédito parcial (acerto de um ou dois atributos), o desempenho de W ellington foi melhor já na fase SAJ $(0,77)$, mantendo o mesmo percentual na fase mais complexa (TRA), onde há blocos superpostos. A análise do desempenho, comparado ao perfil dos demais indicadores sugeriu quea criança, ao diminuir os comportamentos efatores afetivo-motivacionais não-facilitadores, apresentou percepção clara e conduta comparativa (in put), mas teve dificuldade paraintegrar todas as informações para correta solução (elaboração eoutput). Dados sumarizados deWellington no CATM podem ser visualizados naTabela 1.

\footnotetext{
${ }^{9} \mathrm{~N}$ ão havia normas específicas para limitar o teto dedicas; ainda, a criança fazia muitas interrupções, sendo necessário retomar o mesmo nível deajuda. No pós-testefoi estabel ecido o número máximo de 5 dicas para conduzir a criança à solução analógica correta, sendo o último nível deajuda classificado como modelo total.
} 
Tabela 1 - Freqüência e proporção decréditos total e parcial deW ellington nas fases sem ajuda (SAJ), manutenção (MAN) e transferência (TRA) do CATM.

\begin{tabular}{|c|c|c|c|c|c|c|c|c|c|}
\hline \multirow{3}{*}{$\begin{array}{l}\text { Acertos } \\
\text { Crédito }\end{array}$} & \multicolumn{6}{|c|}{ FASES } & \multicolumn{2}{|c|}{ Perfil } & \multirow{3}{*}{$\frac{\text { Taxa de ganho }^{\mathrm{a}}}{\text { SAJ-MAN }}$} \\
\hline & \multicolumn{2}{|c|}{$\begin{array}{c}\text { SAJ } \\
\text { (6 cartões) }\end{array}$} & \multicolumn{2}{|c|}{$\begin{array}{c}\text { MAN } \\
(6 \text { cartões) }\end{array}$} & \multicolumn{2}{|c|}{$\begin{array}{c}\text { TRA } \\
\text { (10 cartões) }\end{array}$} & \multirow[t]{2}{*}{ SAJ-MAN } & \multirow[t]{2}{*}{ SAJ-TRA } & \\
\hline & $\mathrm{F}$ & $\mathrm{P}$ & $\mathrm{F}$ & $\mathrm{P}$ & $\mathrm{F}$ & $\mathrm{P}$ & & & \\
\hline Total & 2 & 0,33 & 3 & 0,50 & 5 & 0,25 & Ganhador & $\begin{array}{l}\text { Não } \\
\text { transferidor }\end{array}$ & 0,51 \\
\hline Parcial & 14 & 0,77 & 13 & 0,72 & 40 & 0,67 & - & - & $-0,06$ \\
\hline
\end{tabular}

Legenda: F-Freqüêcia; P-Proporção. Crédito total máximo nas fases SAJ e MAN =6; TRA $=20$; Crédito parcial máximo nas fases SAJ e MAN $=18 ;$ TRA $=60$; aindicador de mudança $=30 \%$.

Igor, apesar de semelhante perfil nos instrumentos psicométricos, recusou-sea prosseguir na tarefa não completando a fase deASS. A inacessibilidade à mediação foi o componente afetivo-motivacional prevalente, na medida em que a criança recusava-sea aceitar queal gumas respostas não eram corretas. Tentativas do mediador para quea criança persistisse na tarefa o levavam a comportamentos quedenotavam cansaço ou esquiva.

Devido ao severo comprometimento motor deG uilherme (dificuldade para manusear os blocos), real izamos modificações na aplicação do CATM para quepudesseindicar qual figura deveria ser selecionada pela examinadora. Contudo, a dificuldade de retenção dos atributos na memória de trabalho, acrescida da dificuldade de sinalizar "sim" e "não", inviabilizaram a continuidade do teste. A pesar das limitações, a criança conseguiu classificar os blocos na matriz (fase PRE), após serem colocados em sua mão, participando com interesse e demonstrando satisfação aos feedbacks queindicavam bom desempenho.

As demais apresentaram déficits que impediram a compreensão ou recusa para a realização da prova assistida, ainda em suas fases iniciais (impulsividade, método detrabal ho confuso ao abordar a tarefa, seguidos de baixa tolerância à frustração).

\section{O programa de intervenção em CAA}

O programa de intervenção com sistemas de CAA foi conduzido em um período de 3-4 meses, em sessões individualizadas, totalizando 121 sessões (em média, 17,5 sessões/ criança), além de 32 sessões de observação em atividades escolares.

A intervenção foi pautada nos componentes daEAM (FEUERSTEIN et al., 1987; Lidz, 1991) sumarizada nas mediações para: selecionar estímulos, estabelecendo uma ordem em termos de relevância; conduzir a criança à habilidade deplanejamento, mediantecomportamento deauto-regulação; ajudar na aquisição das noções de tempo e espaço; viabilizar a interpretação através do pensamento 
indutivo e/ ou lógico; e ajudar o mediado a transcender as noções adquiridas, ultrapassando o "aqui e agora" da situação imediata (GOMES, 2002; TZURIEL; HAYWOOD, 1992). Nesse sentido, a intervenção, mediada pelo sistema computadorizado Imagoanavox, se traduziu em estratégias para fomentar a elaboração de seqüências comuni cativas mais adequadas, extensas e com funções diversas, a partir da situação de conversação livre ou narrativa estimulada pelos materiais selecionados.

A análisedos indicadores descritos no prétesteforneceu subsídios para a organização dessa etapa. Para quatro crianças queapresentaram mais indicadores facilitadores foram realizadas, al ém das tarefas comunicativas, tarefas cognitivas utilizando jogos ou provas assistidas. Para as demais, com dificuldades de autoregulação e processamento cognitivo, foram realizadas somente as tarefas comunicativas mediadas pelo computador. Mas val e ressal tar que os sistemas de CAA utilizados (Imagoanavox eFichário decomunicação), dada a sua configuração, permitiram trabal har o raciocínio categorial, mediantea seleção deitens agrupados em classes semânticas como pessoas, animais, coisas que faço (verbos), como as coi sas são (adjetivos), entreoutras.

O perfil comunicativo dos participantes durantea intervenção foi obtido mediante a análise dos episódios de emissões comunicativas significativas na interação mediador-criança, no contexto específico da tarefa. Considerando a totalidade das sessões, observou-se maior proporção de emissões do tipo verbal (41\%), gestual (32\%) esimbólica/ mista (18\%), independente de a tarefa principal ser computadorizada ou não. Esta última topografia descrevia as comunicações com o Imagoanavox, de forma exclusiva ou combinada com outras modal idades (palavras, sentenças, vocal izações, gestos e expressão facial ). Quanto à extensão, as emissões variaram de 1-10 unidades (palavras, gestos e/ ou símbolos), apresentando a maioria 1 unidade (69\%), seguida de 2 (18\%) e 3 unidades (7\%). Por fim, a anál ise da função comunicativa indicou uma maior freqüência derespostas (64\%), ou seja, emissões que se seguiam imediatamente às solicitações da mediadora. Comunicações mais espontâneas (que não eram imediatamente precedidas por questões ou mandos) foram classificadas na categoria comentário (23\%), quando a criança fornecia informações sobre diferentes temas (fim de semana, pessoas queridas, atividades realizadas, etc.).

Em geral, o comportamento das crianças nessa fase foi facilitador ( $\mathrm{Md}$ $=0,68)$, mostrando-se relaxadas $(0,81)$, participativas $(0,77)$ e orientadas durante as tarefas $(0,72)$. Foi semelhante a proporção média dos fatores afetivomotivacionais $(M d=0,70)$, com destaque para os itens acessibilidade à mediação $(0,75)$, controleda atitudedefensiva $(0,72)$, além denecessidade deexplorar a tarefa $(0,70)$, curiosidade $(0,70)$ e vitalidade e sentido de alerta $(0,70)$. Em termos de operações cognitivas ( $\mathrm{Md}=0,64)$, as dificuldades envolviam a identificação de relevância $(0,59)$, a generalização $(0,59)$ ea dificuldade de autocorreção $(0,40)$. 
Para obter uma avaliação mais pormenorizada sobre o processo ocorrido na intervenção, foram anal isadas 5 do total de sessões real izadas por cada criança (em média, 17,5), equivalentes à primeira (0), à última (1) e à sessão intermediária (1/2), cal culando-se também as sessões que correspondiam a $1 / 4$ ea $3 / 4$ desse total. O Gráfico 1 apresenta as proporções do grupo para cada um dos indicadores - cognitivo, comportamental e afetivo-motivacional - nessa amostra de 5 sessões, com um padrão semelhante para os comportamentos e operações cognitivas facilitadoras, apresentando os fatores afetivo-motivacionais uma curva menos acentuada.

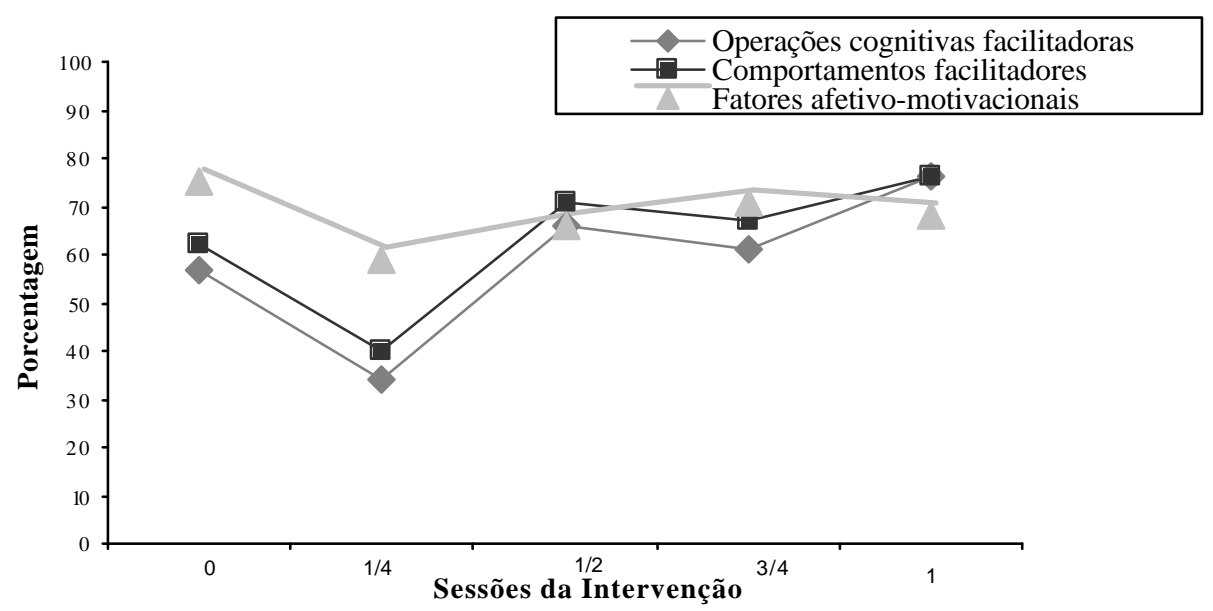

Gráfico 1 - Proporção dos indicadores cognitivos e comportamentais facilitadores e fatores afetivo-motivacionais durante a intervenção no grupo (amostra de 5 sessões).

Considerando a diferença entre os dados da primeira eda última sessão no grupo, houve uma taxa de ganho de 33\% para as operações cognitivas facilitadoras e $23 \%$ para os comportamentos mais positivos. Contudo, houve redução nos fatores afetivo-motivacionais (-9\%). Dados qual itativos esclarecem as diferenças encontradas nesses dados. Na última sessão foi aplicado o Jogo de Analogia, cuja fase de ASS apresentou maior freqüência de fatores afetivomotivacionais não-facilitadores à tarefa, o que rebaixou o desempenho neste indicador.

A despeito da extensão reduzida da maioria dos episódios comunicativos (1 palavra/ símbolo), construídos com o uso do computador, sem o apoio de um sistema deCAA, um interlocutor não familiarizado com a experiência prévia da criança teria extrema dificuldade para compreender toda a narrativa, gerando frustração para ambos na interação. Destaca-setambém a general ização dehabilidades comunicativas. Primeiro, foram signicativos os episódios decrianças como Guilherme, M arcus, Fabrícia e Fernanda em interação com professores e 
familiares, no início ou final da sessão, utilizando o Imagoanavox para estabelecer comunicação significativa efuncional. Em segundo lugar, houveo relato denovas palavras, inseridas no sistema deCAA, nos contextos da casa esala deaula.

\section{Resultados do Pós-TESTE}

Os instrumentos psicométricos e a prova assistida foram reaplicados no pós-teste. Para a maioria, não ocorreu mudança nas classificações das provas psicométricas cognitivas, mas houve pequena melhora na capacidade de compreensão da linguagem, medida pelo resultado no TVIP. A menor freqüência de comportamentos de esquiva permitiu que al gumas crianças persistissem com mais interesse e motivação nas mesmas provas computadorizadas (Columbia e TVIP). O Gráfico 2 apresenta o aumento na proporção de comportamentos facilitadores para todas as provas psicométricas.

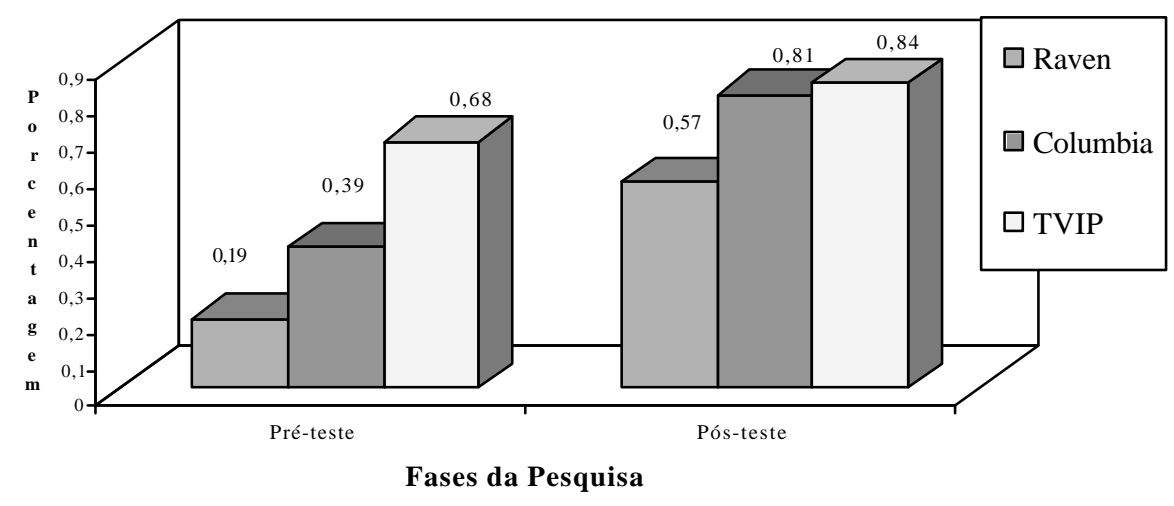

Gráfico 2 - Proporção de comportamentos facilitadores da amostra nas provas psicométricas, no prée pós-teste $(\mathrm{N}=7)$.

No tocante a execução do CATM, as crianças apresentaram um desempenho um pouco mel hor após a intervenção. Em relação à avaliação no pré teste, duas crianças conseguiram completar todas as 5 fases da prova etrês crianças com maior déficit cognitivo e problemas comportamentais conseguiram realizar a fase PRE (completar a matriz de blocos segundo os atributos). Ainda nessa fase, considerando-se a proporção média de acerto total (sem ajuda), entre o pré e o pós-teste, seis crianças melhoraram o desempenho, e a taxa de ganho do grupo ficou em $67 \%$. Semel hante à análise nas provas psicométricas pode-se dizer que com a redução de comportamentos não-facilitadores e maior acessibilidade às estratégias de mediação o grupo persistiu um pouco mais na prova assistida.

Uma análise mais específica dos dados de Wellington e Igor que realizaram integral mentea prova assistida na faseem questão mostrou que, apesar 
da melhora em alguns indicadores, quando se compara o pré e o pós-teste, eles não foram capazes de manter as estratégias de resolução analógica após a suspensão da ajuda (percentual mínimo de 0,60 para acerto total comparando-seas fases SAJMAN). Ambas as crianças apresentaram perfis semel hantes quanto aos 3 indicadores considerados, iniciand o bem a fase PRE, mas demonstrando um padrão de diminuição mais evidente na fase de ASS, quando apresentaram baixo escore no fator trabal har de forma independente, seguido de interesse em explorar a tar efa e prazer na tarefa. Considerando os 3 indicadores, W el lington apresentou índicesmais facilitadores do quel gor. E quando a anál ise incidiu sobreos créditos parciais, foi possível constatar uma taxa de ganho de ganho de 0,63 para W ellington entre as fases SAJ eTRA (mais complexa).

\section{Discussão}

Como vimos, no pré-teste, o baixo desempenho nas habilidades medidas nas provas psicométricas foi em parte afetado pela alta freqüência de comportamentos não-facilitadores, que também prejudicaram a realização e o desempenho na prova assistida. A pós a intervenção, de modo geral, as crianças foram mais persistentes e concentradas nas tarefas computadorizadas (Columbia eTVIP), apresentando interesseecuriosidade para explorar as telas eestabelecendo relações entre as figuras estímulos e seus referentes, com a expressão de gestos, nomeações ecomentários. Assim, asmesmas tarefas computadorizadas setornaram mais motivadoras, quando comparamos o prée o pós-teste. Esses dados sugerem que estratégias mediacionais, especialmente, o incentivo à auto-regulação e a significação da tarefa (implementadas durante a intervenção), podem ter contribuído para uma maior participação do grupo.

O desenvolvimento de provas computadorizadas ainda é um campo pouco explorado na avaliação assistida. Analisando o desempenho de quatro partici pantes nas tarefas cognitivas mais estruturas como a Prova deExclusão de Objetos e o Jogo de A nalogia de Figuras, uma alternativa seria a ampliação destes instrumentos (para melhor composição das fases com e sem ajuda) em versão computadorizada. Cada problema, sendo formado por diferentes figuras coloridas e organizado com poucos itens, pode ser apresentado em telas sucessivas, o que viabilizaria que crianças com sério comprometimento motor real izassem a tarefa (como éo caso deGuilherme), sendo mais motivador para outras.

Entre os aspectos positivos na prova assistida, o suporte instrucional, por exemplo, foi mais expressivo para W ellington, pois melhorou o desempenho, quando se comparam as fases antes e após a assistência no pré-teste. Semelhante ao estudo com o CATM em amostra brasileira (SANTA MARIA; LINHARES, 1999), foi possível verificar as operações cognitivas e as disfunções nas diferentes fases da prova, bem como as variáveis comportamentais que afetaram as respostas, compondo um perfil de aprendizagem na situação de problemas de raciocínio analógico. 
A identificação dos padrões cognitivos e não-intelectuais nos participantes sugerem, a partir daquelas já propostas (SANTA MARIA; LINHARES, 1999), a necessidade de revisão enovas adaptações na extensão da prova assistida, visando sua adequação e uso em crianças com déficits de comunicação. Sugere-se ainda adequar o procedimento de níveis de ajuda estruturado, proposto por CampioneeBrown (1987) para outras crianças com perfil semelhanteao daamostra. Esteéapresentado de forma seqüencial: inicia-secom o nível menor deassistência (instrução), seguindo atéa ajuda total (modelo), sempreajustado à necessidadeda criança, para modificar a forma deapreender os estímulos. No prétestedo CATM, devido aos comportamentos não facilitadores, era necessário iniciar com o nível 2 deajuda (nomear os atri butos dos três primeiros blocos eentão indagar "C om qual bloco el evai formar par?" ) . Isto ajudava a criança a retornar a atenção para o problema. Já no pós-teste, com a diminuição decondutas dispersivas, a assistência iniciou-se no nível 1 deajuda (repetir a instrução sem nomear os atributos dos três primeiros blocos). É possível que crianças com déficits de atenção e memória necessitem começar a partir desse nível 2 de ajuda, que seria utilizado como instrução básica para todas as fases da prova assistida, mesmo as sem ajuda. Dados semel hantes foram encontrados no procedimento assistido aplicado por Enumo eBatista (2000) em crianças com deficiência visual, particularmenteas com baixa visão grave. Tais crianças precisaram de uma assistência básica para a resol ução, mesmo nas fases ondea ajuda do examinador deveria ser suspensa. Nesse sentido, os resultados do subgrupo de crianças com maior comprometimento na capacidade visual se assemel haram ao nosso no tocanteàs categorias comportamentais (cansaço, baixa concentração e queda de desempenho) em determinadas fases e quanto ao nível deajuda necessário.

A pesar das limitações assinal adas, os instrumentos utilizados no préteste forneceram indicações para planejar a intervenção em tarefas lingüísticocognitivas, mediadas pelo computador. Segundo Fonseca (1998), as disfunções cognitivas da atenção, percepção, sel eção eorganização da informação podem ser modificadas estrutural mente, por meio desituações mediadas mais intencionaise motivadoras que encorajem a criança a se adaptar a novas e diferentes situações deaprendizagem. Por mais queocorram limitações impostas pela deficiência, cada indivíduo possui uma possibilidade de mudança cognitiva que não pode ser negligenciada. A té mesmo V inícius, que não apresentou indicador de mudança nas operações cognitivas entre o pré e o pós-teste, obteve ganho, quando consideramos o repertório comuni cativo e o padrão de interação com o mediador na fase de intervenção (constante nas três últimas sessões): começou a utilizar o sistema deCAA de forma mais efetiva nas comunicações queenvolviam solicitações e respostas a questões diferentes de $\mathrm{sim} /$ não, aumentando o número de palavras e a emissão de sentenças mais significativas.

Pode-se dizer que o perfil da maioria do grupo (baixa tolerância à frustração e reduzida auto-regulação), identificado nas fases deavaliação inicial e préteste do procedimento assistido, pode ser considerado semelhante ao de 
crianças normais, em idade pré-escolar, cujas operações cognitivas possivel mente estão em processo de maturação (TZURIEL, 2001). Desse modo, mesmo a fase preliminar (PRE) deve ser sobremodo val orizada na avaliação de crianças com NEE. N o caso, foi preciso elaborar um protocolo mais específico do desempenho, se constituindo em uma etapa de ensino voltada para a classificação correta dos atributosnamatriz.

A capacidade de raciocinar por analogias é uma das habilidades mais complexas no desenvolvimento das funções cognitivas superiores, em queseextrai um novo significado ou informações daquilo que é percebido ou conhecido (AN GELINI et al., 1999). Comparando os instrumentos queavaliaram o raciocínio analógico, o Raven-MPC foi a prova de maior dificuldade, e como produto, foi possível identificar déficits nessa importantehabilidadesuperior. O CATM, apesar detodas as limitações já discutidas, permitiu de forma mais abrangente, identificar aspectos do processo de resolver problemas analógicos. Na diminuição da conduta impulsiva, a criança buscava, entreas al ternativas, a solução correta, e setornava mais acessível à mediação, ainda que o fator motivação intrínseca à tarefa permanecesse baixo na fase deASS.

Ainda sobre os dados da avaliação assistida, estudos têm apontado que o procedimento dinâmico permite identificar variações intragrupo, mesmo quando os sujeitos estão equi parados nas classificações psi cométricas (ENUMO, 2005; ENUMO; BATISTA, 2000; SANTA MARIA; LINHARES, 1999). Foi possível observar as diferenças no perfil de desempenho entre Igor e Wellington e as estratégias mediacionais mais expressivas para este, levando a um aumento no número deacertos no crédito total, no pré-teste. Comparando-se a fasecom maior nível dedificuldade (transferência-TRA) no préeno pós-teste, W ellington manteve a proporção deacertos no crédito parcial (SAJ-MAN-TRA).

Um outro aspecto que se destaca na avaliação assistida éa possibilidade de consi derar o contexto na situação de aval iação, diferentemente do que ocorre com os testes convencionais queassumem que os indivíduos operam em ambientes descontextualizados (STERNBERG; GRIGORENKO, 2002). Nesse aspecto, o desempenho deW ellington no CATM (mais rebaixado no pós-teste) podeter sido, em parte, influenciado por aspectos contextuais. No final do período escolar, a criança apresentou um padrão geral de falta de motivação, preferindo dormir e brincar em casa. Estas informações relatadas pela mãe eeducadoras coincidiram com o ingresso da criança em instituição especial de reabilitação que, via de regra, insere a criança em vários atendimentos especializados, paral elos à atividade escolar. A ssim, é importante identificar, em um processo de avaliação, os fatores que afetam as relações entremediação emotivação para apren der.

Como são poucos os estudos que descrevem o grau de influência dos fatores afetivo-motivacionais na performance da criança na situação detestagem, há necessidade de estabel ecer a confiabilidade na definição de cada um de seus componentes. Pesquisas têm encontrado baixos índices deconcordância entrejuízes 
na avaliação dos componentes da avaliação assistida (TZURIEL; SAMUELS, 2000; VAUGHT; HAYWOOD, 1990). A exemplo da pesquisa deTzuriel eSamuels (2000) que obteve níveis baixos de confiabilidade em determinados fatores afetivomotivacionais, nossa análise desses indicadores por fase, indicou que os componentes tol erân cia à frustração eatitu de defensiva apresentaram os índices de concordância mais baixos; já os componentes trabalhar de forma independente e vital idadee sentido de al erta obtiveram escoremédio. Esses dados sugerem queainda há pouca clareza nas definições de tais categorias. Um outro fator que pode ter contribuído para o baixo índice pode ter sido uma al ta flutuação da criança entre os indicadores (por exemplo, alternava comportamentos facilitadores e não facilitadores), em uma mesma fase, elevando o nível de interpretação divergente entre os juízes. Contudo, categorias como acessibilidade à mediação, necessi dade de explorar a tarefa eprazer na atividade apresentaram al to índice deconcordância. Dada a relevância do tema, a influência dessas variáveis no uso do procedimento assistido está sendo considerada em novos estudos (ENUMO et al, 2003).

A inda sobreos fatores não-intelectuais, a superexposi ção a programas de estimulação pode levar a criança a desenvolver estratégias que a tornam, de certo modo, "imune" à mediação do adulto (TZURIEL, 2001). I gor pareceretratar essecaso clássico descrito pelo autor. Quando pequeno, a criança foi submetida a um exaustivo programa de intervenção precoce. Em geral, em situação de aprendizagem, demonstrava um padrão de controle rígido sobre o queaprender e quando aprender, se esquivando de tarefas escolares mais estruturas. Embora pudesse "navegar" entre os módulos do programa computadorizado - imagem e anagrama (letras egrupos silábicos) - apresentou pouca motivação para explorar relações entrefiguras ealfabeto. Observações em sala eno laboratório, ao longo da intervenção, indicaram o mesmo perfil deaprendizagem, ou seja, menor interesse para atividades de leitura e escrita. O mesmo perfil foi observado em W ellignton, apesar de trajetória diferente em serviços de estimulação. Desse modo, ambas as crianças, em situação de ensino, eram comumentes descritas pelas professoras como alunos sem muita motivação para aprender.

A motivação é uma questão sobremodo relevante e uma das mais desafiadoras para a educação inclusiva. Conformeaponta Sternberg e Grigorenko (2002), este construto deveser considerado como o componenteindispen sável para o sucesso escolar. Sem ela, o estudantenão terá iniciativas para aprender. Em outras palavras, éa motivação que ativa as habilidades metacognitivas, além das funções cognitivas que envolvem a aprendizagem. Assim, da análise aqui depreendida sobre os fatores afetivo-motivacionais, consideramos que os procedimentos da avaliação assistida e da CAA podem se constituir em importantes ferramentas conceituais para a elaboração deestratégias que viabilizem a motivação do aluno para aprender.

Sobreo processo deinclusão éinteressante considerar que, na amostra de setecrianças, apenas uma estavainserida no sistema deensino particular (daquelas que freqüentavam a escola pública, apenas uma era proveniente de nível sócio- 
econômico mais baixo). Para a maioria das mães, a escola públicaéa quetem oferecido um ensino mais ad equado às necessidades educativas deseus filhos, considerando, ainda, que as crianças participavam bem das atividades em grupo, de eventos comemorativos e outros programas escolares. Algumas relataram que a maior dificuldade ocorria nas interações sociais fora do ambiente escolar, com pares desconhecidos, onde surgiam sinais de pouca tolerância para determinados comportamentos da criança. Este relato corrobora dados de estudos mais recentes onde já se começa a vislumbrar que, de um modo geral, as experiências inclusivas da criança com deficiência no ensino regular estão apresentando resultados positivos (BATISTA; ENUM O, 2004; STAINBACK; STAINBACK, 1999; VON TETZCHNER et al., 2005), especialmente, no tocanteà interação desses alunos com pares diferentes, possivel mente contribuindo para a diminuição de preconceitos e de condições sociais estigmatizantes (OMOTE, 2004). Contudo, é preciso considerar se, além das habilidades sociais, as demais necessidades espeć́ficas deaprendizagem decrianças com deficiência, particilarmenteno quese refereà competência comunicativa, estão sendo atendidas, e se há a introdução da CAA no currículo escolar, de maneira sistematizada, em todas as regiões do país.

Embora as consi derações aqui real izadas não possam ser transpostase general izadas para outros grupos de crianças com problemas de comunicação, devido ao reduzido tamanho da amostra (MELTZOFF, 2002), a avaliação, na modalidade tradicional, permitiu comparar o desempenho de crianças com problemas de comunicação com seus pares e a modalidade assistida permitiu mapear importantes indicadores no desempenho do grupo, bem como características individuais que ajudaram a direcionar o programa de intervenção. Com isso, espera-se que os resultados apresentados contribuam para a discussão de questões metodológicas sobre a adequação e uso de instrumentos de aval iação e ensino em outras populações, assim como para ampliar o reduzido número de estudos (CARRILLO; TH OM PSON, 2002) que unem as recentes áreas da CAA e da avaliação assistida. A inda temos poucos estudos quem enfocam uma avaliação mais estruturada em crianças com desenvolvimento mais cromprometido.

\section{Considerações finais}

A creditamos quea área da avaliação edemedidas psicológicas, desde o tempo das primeiras aplicações dos testes deinteligência por Binet, amadureceu o suficiente para não incorrer novamente no grave erro de contribuir para os processos deexclusão eestigmatização dos quepossuem al guma deficiência. Vale ressaltar mais uma vez queas avaliações são importantes quando contribuem para o desenvolvimento de estratégias adequadas que melhorem as condições psicológicas, educacionais, afetivas esociais denossas crianças ejovens.

Seguindo nessa linha, as pesquisas sobre a avaliação assistida, nos últimos anos, vêm del ineando um campo bastantepromissor dada as características inovadoras que a distinguem de uma avaliação tradicional, beneficiando a 
população queapresenta déficits no processo de aprendizagem ou queseencontra em situação de desvantagem social. Variações intragrupo na população especial são consideradas, permitindo a el aboração deintervenções individual izadas.

A pesar das limitações apontadas, mesmo os críticos daárea a descrevem como umaw on derful idea (STERN BERG; GRIGORENKO , 2002), dada a possibilidade de fornecer informações mais úteis e vál idas para pais, educadores e psicólogos, particularmentenos campos da Educação Especial, Psi cologia clínica e Psicologia do desenvolvimento.

Mesmo nessa atmosfera otimista, há muito por fazer, especialmente em nosso país, onde os estudos empíricos desenvolvidos a partir da década de 90 ainda estão restritos a poucos grupos de pesquisa. Nesse campo mais geral da avaliação psicológica e educacional, a aval iação assi stida caminha no sentido de adquirir o status e o rigor científicos já conquistados pela abordagem psicométrica durante o curso do último século. Assim, esperamos ter al cançado, ao final dessas apreciações, a nossa proposta principal: a de divulgar tão relevante temática e, possivelmente, suscitar a curiosi dade e o interesse de outros pesquisadores, para que possam, talvez, incrementar a área com futuras investigações. Em outras palavras, aguardamos novos tempos no campo da avaliação psicológica e seu impacto direto nas medidas educacionais para uma sociedade mais inclusiva. A integração de áreas tão promissoras, como a avaliação assistida ea CAA , poderá auxiliar no desenvolvimento de intervenções psicológicas mais sensíveis eeficazes, contribuindo para melhorar a qualidade de vida das pessoas com deficiência e outras necessidades específicas de ensino.

\section{Referências}

ANGELINI, A.L. et al. M anual M atrizes Progressivas Coloridas de Raven: escala especial. São Paulo: Centro Editor de Testes e Pesquisas em Psicologia, 1999.

BATISTA, M.W.; ENUMO, S. R. F. Inclusão escolar edeficiência mental: análise da interação social entre companheiros. Estudos de Psicologia, v. 9, n. 1, p. 101-111, 2004.

BATISTA, C.G.; HORINO, L.E.; NUNES, S.S. A valiação assistida de habilidades cognitivas em crianças com deficiência visual e com dificuldades deaprendizagem. P sicologia: R eflexão eCrítica, v. 17, n. 3, p. 381-393, 2004.

CAMPIONE, J.; BROWN, A.L. Linking dynamic assessment with school achievement. In: LIDZ, C.S. (Ed.). D ynamic assessment: an interactional approach to evaluating learning potential. New York: The Guilford Press, 1987, p. 82-115.

CA POVILLA, F.C.; CA POVILLA, A.G.S. Desenvolvimento lingüístico na criança dos dois aos seis anos: tradução e estandardização do Peabody Picture Vocabulary Test de Dunn \& Dunn, e da Language Development Survey de Rescorla. Ciência Cognitiva: Teoria P esquisa e A plicação, v. 1, n. 1, p. 353-380, 1997.

CAPOVILLA, F.C.; DUDUCHI, M.; MACEDO, E.C. M TS - Programa computadorizado para criação de provas de avaliação cognitiva e de experimentos em P sicologia [CD ROM]. São Paulo: QS Informática S/ C Ltda, 1997a 
CAPOVILLA, F.C.; DUDUCHI, M.; MACEDO, E.C. M anual do Sistema Computadorizado ImagoA naV ox [CD ROM]. São Paulo: QS Informática S/ C Ltda, 1997b.

CAPOVILLA, F.C. ; THIERS, V.O. ; CAPOVILLA, A.G.S. A valiação cognitiva de crianças com severos distúrbios motores: versões computadorizadas, normatizadas e validadas de cinco testes de vocabulário, compreensão auditiva, prontidão para leitura e inteligência geral. In: NUNES, L.R.O.P. (Org.). Favorecendo o desenvolvimento da comunicação em crianças e jovens com necessidades educacionais especiais. Rio de Janeiro: Dunya, 2003, p. 253-262. CARRILO, M.; THOMPSON, B. Communication needs of school-aged low verbal/ nonverbal children. In: CONFERENCE OF THE INTERNACIONAL SOCIETY FOR AUGMENTATIVE AND ALTERNATIVE COMMUNICATION (ISAAC), $X, 2002$, Denmark. Proceedings... Denmark: ISAAC, 2002, p. 58-59.

DIAS, T.L.; ENUMO, S.R.F. Criatividade em crianças com dificuldade de aprendizagem: A valiação e intervenção através de procedimentos tradicional e assistido. Psicologia: Teoria e Pesquisa, Brasília, DF, v. 22, n. 1, p. 69-78, 2006.

DIAS, T.L. et al . A valiação assistida: contribuições a programas de intervenção para crianças com necessidades educativas especiais. In: ALCHIERI, J.C.; CRUZ, R.M. (Org.). A valiação psicológica no contexto iberoamericano. Porto A legre: Artmed, no prelo.

EN UM O, S.R.F. A valiação assistida para crianças com necessi dades educacionais especiais: um recurso auxiliar na inclusão escolar. Revista Brasileira de Educação Especial, Marília, v. 11, n. 3, p. 335-354, 2005.

ENUMO, S.R.F.; BATISTA, C.G. Evaluation of cognitive abiliteis of visually impaired children. In: STUEN, C. et al. (Eds.). Vision rehabilitation: assessment, intervention and outcomes. New York: Swets \& Zeitlinger, 2000, p. 379-381.

ENUMO, S.R.F. et al. Comportamentos do mediador e da criança com deficiência visual na aval iação assistida de habilidades cognitivas. Temas em Psicologia, v. 10, n. 1, p. 71-84, 2002.

ENUMO, S.R.F. et al. Influências de variáveis mediadoras do desempenho cognitivo, lingüístico, matemático e criativo em intervenções e provas assistidas para crianças com necessidades educativas especiais. Projeto de pesquisa CN Pq n 501014/ 2003-9. Vitória, ES: UFES, 2003.

FEUERSTEIN, R. et al. Prerequisites for assessment of learning potential: the LPAD model. In: Lidz, C. S. (ed.), Dynamic A ssessment: an interactional approach to evaluating Learning Potential. London: Guilford Press, 1987, p. 35-51.

FONSECA, V. A prender a aprender: a educabilidade cognitiva. Porto Alegre: Artmed, 1998. GOMES, C.M .A. Feuerstein e a construção mediada do conhecimento. Porto Alegre: Artmed, 2002.

HAYWOOD, H.C.; TZURIEL, D. Applications and challenges in dynamic assessment. Peabody Journal of Education, v. 77, n. 2, p. 40-63, 2002.

LIDZ, C.S. Practioner's guide to dynamic assessment. New York: The Guilford Press, 1991.

LINHARES, M.B.M.; ESCOLANO, A.A.C.M.; ENUMO, S.R.F. (Org.). A valiação cognitiva assistiva: fundamentos, procedimentoseaplicabilidade. São Paulo: Casa do Psicólogo, 2006.

LINHARES, M.B.M. et al. Avaliação assistida: uma abordagem promissora na avaliação cognitiva de crianças. Temas em Psicologia, v. 6, n. 3, p. 231-254, 1998.

MELTZOFF, J. Critical thinking about research: Psychology and related fields. Washington, DC: American Psychological Association, 2001. 
NUNES, L.R.O.P. (Org.). Favorecendo o desenvolvimento da comunicação em crianças e jovens com necessidades educacionais especiais. Rio de Janeiro: Dunya, 2003.

NUNES, L.R.O.P. et al. Ampliando o uso da comunicação al ternativa: procedimentos para expansão da narrativa. In: GONÇALVES, M.J. et al. (Org.). Tecnologia em (re)habilitação cognitiva. São Paulo: Centro Universitário São Camilo, 2000, p. 275-282.

OMOTE, S. Estigma no tempo da inclusão. Revista Brasileira de Edu cação Especial, Marília, v. 10, n. 3, p. 287-308, 2004.

PAULA, K.M.P. D esenvolven do a linguagem funcional em criança com deficiência múltipla através de sistemas de comunicação alternativa e do ensino naturalístico. 1998. 163p. Dissertação (Mestrado em Educação) - Universidade do Estado do Rio de Janeiro, Rio de Janeiro.

. A valiação A ssistida: Análise de indicadores cognitivos, comportamentais e afetivomotivacionais em crianças na situação de intervenção com sistema de comunicação alternativa. 2004. 288p. Tese (Doutorado em Psicologia) - Universidade Federal do Espírito Santo, Vitória, 2004.

PAULA, K.M.P.; ENUMO, S.R.F. A valiação cognitiva assistida na situação de intervenção com sistemas de CAA. In: NUNES; L.R.O.P.; PELOSI, M.B.; GOMES, M .R. U m retrato da pesquisa em Comunicação A Iternativa no Brasil. Rio de Janeiro: EDUERJ, no prelo.

RODRIGUES, A.; ROCHA, J.M. Escala Columbia de M aturidade Intelectual: manual de aplicação. Rio de Janeiro: CEPA, 1999.

SANTA MARIA, M.R.; LINHARES, M.B.M. A valiação cognitiva assistida de crianças com indicações de dificuldades de aprendizagem escolar e deficiência mental leve. Psicologia: Reflexão e Crítica, v. 12, n. 2, p. 395-417, 1999.

STAIN BACK, S.; STAINBACK, W. (Org.). Inclusão: um guia para educadores. Tradução de M. F. Lopes. Porto Alegre: ArtMed., 1999.

STERNBERG, R.J.; GRIGORENKO, E.L. Dynamic Testing: the nature and measurement of learning potential. N ew York: Cambridge University Press, 2002.

TZURIEL, D. D ynamic assessment of young children. New York: Kluwer Academic/ Plenum Publishers, 2001.

TZURIEL, D.; HAYWOOD, H.C. The development of interactive-dynamic approaches to assessment of learning potential. In: HAYWOOD, H. C., TZURIEL, D. (Eds.). Interative assessment. N ew York: Springer-Verlag, 1992, p. 3-37.

TZURIEL, D.; KLEIN , P.S. The Children's A nalogical Thinking M odifiability Test: instrucion manual. Ramat-Gan: School of Education Bar Ilan University, 1990.

TZURIEL, D.; SA MUELS, M.T. Dynamic assessment of learning potential: inter-rater reliability of deficient cognitive functions, types of mediation, and non-intellective factors. Journal of Cognitive Education and Psychology, v. 1, p. 2-23, 2000. Disponível em: বhttp:/ / www.iacep.coged.org>.

TZURIEL, D.; SAMUELS, M.T.; FEUERSTEIN, R. Non-intellective factors in dynamic assessment. In: GUPTA, R.M., COXHEAD, P. (Eds.). Cultural diversity and learnnig efficiency: recent developments in assessment. London: NFER-N elson, 1988, p. 141-163.

VAUGHT, S.R.; HAYWOOD, H.C. Interjudge agreement in dymanic assessment: two instruments form Learning Potential Assessment Device. The Thinking Teacher, v. 5, p. 2-6, 1990. 
VON TETZCHNER, S. et al. Inclusão decrianças em educação pré-escolar regular utilizando comunicação suplementar e alternativa. Revista Brasileira de Educação Especial, Marília, v. 11, n. 2, p.151-184, 2005.

VYGOTSKY, L.S. A formação social da mente: o desenvolvimento dos processos psicológicos superiores. Tradução de J. C. N eto, L. S. M. Barreto e S. C. Afeche. São Paulo: Martins Fontes, 1991.

WARREN, D.H. Blindness and children: an individual differences approach. E.U.A.: Cambridge University Press, 1994. 